\title{
Desarrollo tardío del lenguaje: la conciencia metapragmática en la edad escolar"
}

\author{
Late Language Development: Metapragmatic Consciousness \\ in School-Aged Children
}

Recibido: julio 13 de 2009 | Revisado: agosto 13 de 2009 ～Aceptado: septiembre 14 de 2009

\author{
Nina CRESPO-Allende** \\ Pedro Alfaro-Faccio \\ Pontificia Universidad Católica de Valparaíso, Chile
}

* Investigación financiada por FONDECYT 1070333.

** Pontificia Universidad Católica de Valparaíso, Avda. Brasil 2830, 9oㅗ piso, Valparaíso-Chile.

Correos electrónicos: ncrespo@ucv.cl;

RESUMEN

Durante la escolaridad, el lenguaje oral se incrementa tanto cuantitativa como cualitativamente. Entre los cambios cualitativos se encuentra el desarrollo de la conciencia metapragmática que es la capacidad para discriminar y relacionar los estímulos lingüísticos y no lingüísticos. Aunque existen diversas hipótesis para explicar este desarrollo, no hay pruebas empíricas que muestren estos cambios ni señalen las edades fundamentales en las que aparecen. Para la presente investigación, se aplicó un instrumento a 160 estudiantes de 6, 8, 10 y 12 años. Los resultados sugieren que a medida que aumenta la edad, aumentan las respuestas metapragmáticas (conscientes) y disminuyen las epipragmáticas (automáticas). Se encontraron diferencias significativas entre los distintos grupos de edad y se observó que las respuestas dependen del tipo de ejercicio por resolver. Por último, se corroboró que lo metapragmático no es sistemático, su eficacia varía según la forma en que se relacionan el mensaje lingüístico y el contexto.

Palabras claves autores

Conciencia metapragmática, lingüística, escolares.

Palabras claves descriptores

Adquisición del lenguaje, metalinguistica, metacognición, psicolinguistica.

\section{A B S T R A C T}

During schooling, children's oral language skills increase qualitatively and quantitatively. Among the qualitative changes, the development of metapragmatic awareness - the ability to discriminate and to establish relationships between linguistic and non-linguistic stimuli -can be found. Despite the various hypotheses that attempt to account for this development, there is no empirical evidence that might explain these changes or specify the ages at which they appear. For the purpose of this article, a test was administered to 160 children between the ages of 6 and 12 . The results suggest that as age increases, metapragmatic (conscious) responses increase and epipragmatic (automatic) responses decrease. Likewise, significant differences were found among the age groups, and their responses to the test items depended on the type of exercise, thus confirming that metapragmatic skills are not systematic; their effectiveness varies according to the relations between the linguistic message and the context.

Key words authors

Metapragmatic Consciousness, Linguistics, School-Aged Children.

Key words plus

Language Acquisition, Metalinguistics, Metacognition, Psycholinguistics. 


\section{Introducción}

Durante la edad escolar, el lenguaje del niño va enriqueciéndose tanto cuantitativa como cualitativamente (Nippold, 1998). Por tanto, aumenta el vocabulario y las estructuras sintácticas que el niño es capaz de utilizar e interpretar. Asimismo, se da un desarrollo de una capacidad inferencial que le permite, entre otros aspectos, usar adecuadamente formas de lenguaje no literal, como las frases hechas metafóricas y variar su registro de acuerdo con las circunstancias lingüísticas (Ravid $\&$ Tolchinsky, 2002). De manera subyacente a este manejo inferencial, es posible identificar una habilidad más general: la conciencia metapragmática que le permite al sujeto autodirigir el uso comprensivo y expresivo del lenguaje (Gombert, 1992; Verschueren, 2000, 2002).

Varios autores (Gombert, 1992; Puyuelo \& Rondall, 2003; van Kleeck, 1994, 1995) proponen que esta habilidad se desarrolla durante la edad escolar y plantean diversas teorías acerca de cómo se produce este fenómeno. Sin embargo, disponemos de pocos datos empíricos que nos permitan examinar estos cambios en poblaciones representativas de niños hispanohablantes. Por este motivo, el objetivo central del presente trabajo es dar cuenta de las diferencias en conciencia metapragmática que se observan entre niños de distintas edades, por lo que se puede inferir un proceso de desarrollo posterior a los seis años, período en el que aumentan las habilidades metapragmáticas. Primero, se delimitará el concepto de conciencia metapragmática y los modelos que dan cuenta de su desarrollo. Posteriormente, se detallarán y fundamentarán las decisiones metodológicas del trabajo empírico (muestra e instrumento) y se presentará, por último, el análisis estadístico con los principales resultados, que serán discutidos en sus alcances y proyecciones.

\section{Conciencia metalingüística y conciencia metapragmática}

Gombert (1992) y Karmiloff-Smith (1994), entre otros, señalan la existencia de una capacidad que permite regular de manera consciente el uso del lenguaje. Esta capacidad presupone el conocimiento del lenguaje como objeto y se denomina conciencia metalingüística. Al definirla, Gombert (1992) señala que la conciencia metalingüística, esbozada por él, es diferente a la idea de función metalingüística planteada por Jakobson (1963). Para este último, la función metalingüística se relaciona fundamentalmente con el rol que cumple el lenguaje cuando es usado para referirse al lenguaje mismo. Para Gombert, en cambio, los alcances del término tienen un cariz más psicológico, puesto que considera que la conciencia metalingüística forma parte de la metacognición (Flavell, 1993). Dentro de esta perspectiva, la conciencia metalingüística implica, además de actividades de reflexión sobre el lenguaje y de la manera en que este se utiliza, la capacidad del sujeto para controlar y planificar sus propios procesos en el uso comprensivo y expresivo del lenguaje. Así, la conciencia metalingüística se relaciona con el monitoreo cognitivo del lenguaje.

Tanto Puyuelo y Rondal (2003) como FlórezRomero et al. (2006) señalan que existen distintas hipótesis explicativas respecto al desarrollo de las capacidades metalingüísticas. Concretamente, Flórez-Romero et al. señalan dos posturas teóricas diferentes: una que defiende que este desarrollo es producto de la regulación general del sistema cognitivo, y otra que propone que dichas habilidades dependen, en parte, del desarrollo del lenguaje.

En el primer grupo cabe contar con autores como Bialystok (1992) y van Kleeck $(1994,1995)$, quienes han propuesto que los procesos que subyacen a las habilidades metalingüísticas requieren del dominio cognitivo general. Especialmente, van Kleeck (1994) y Levy (1999) se basan en la teoría de Piaget (1987) para explicar la emergencia de la capacidad metalingüística, relacionándola con los estadios de desarrollo cognoscitivo. Para estos autores, el comportamiento metalingüístico evidenciado por los niños está relacionado con la etapa del desarrollo cognitivo en la que se encuentran.

Flórez-Romero et al. (2006) hacen hincapié en dos cualidades, centración e irreversibilidad, que Piaget (1987) atribuye al pensamiento de los niños preescolares, quienes, según esta teoría, se 
encuentran en la etapa preoperacional, es decir, que no han alcanzado el desarrollo de las operaciones lógicas. La centración se entiende como la incapacidad de enfocarse en más de un aspecto a la vez en una situación dada, razón por la cual los sujetos, durante una situación comunicativa, monitorean exclusivamente la fluidez del intercambio para favorecer la comunicación. Por no poder concentrarse en más de un aspecto, en el caso de la comunicación, atienden mayoritariamente al significado, pues sería lo más importante dentro del intercambio. La irreversibilidad, por su parte, se refiere a que los sujetos, en la etapa preoperacional, no pueden entender que ciertas operaciones son reversibles, como por ejemplo la posibilidad de verter agua de un recipiente alto y delgado a uno ancho y bajo, y luego devolverla al primer recipiente manteniendo la misma cantidad de agua (Piaget, 1984). En cuanto al lenguaje, estos autores demuestran que los niños manejan exclusivamente la forma o el significado, y no pueden realizar procesos de reversibilidad que les permitan analizar el discurso del hablante (van Kleeck, 1994). De esta manera, Levy (1999) señala que los menores de seis años muestran un desempeño metalingüístico pobre, porque su forma de pensar les impide separar la forma y el significado del lenguaje en una situación comunicativa, para poder considerar cada elemento por separado. El hecho de que este limitado desempeño metalingüístico empiece a enriquecerse a partir de los seis años, indicaría, según van Kleeck (1994), que los sujetos han pasado a una nueva etapa del desarrollo cognitivo.

Gombert (1992) y Karmiloff-Smith (1994), por otra parte, afirman que el desarrollo metalingüístico depende intrínsecamente del desarrollo dellenguaje, aún cuando para esta última autora la representación interna que se hace el sujeto y el proceso de redescripción representacional que le permite el accesos al nivel metalingüístico, juega un rol fundamental:

El modelo RR [redescripción representacional] postula que las propias representaciones lingüísticas son posteriormente objeto de redescripción, de manera que se convierten en objetos de atención lingüística al margen de su utilización en procesos de comprensión y producción. Las representaciones lingüísticas que se construyen durante la infancia les sirven a los niños para comprender y producir su lengua materna. Pero esas representaciones lingüísticas iniciales no están disponibles como datos para la reflexión metalingüística. Para alcanzar ese objetivo, es preciso que el conocimiento embutido implícitamente en los procedimientos lingüísticos (representaciones del nivel I) se re-represente. (p. 70)

Karmiloff-Smith (1994, p. 71), luego de haber estudiado el desarrollo metalingüístico en infantes francófonos, sostiene "que cada nivel de redescripción representacional no se adquiere a la misma edad. El modelo RR pretende explicar de qué manera se hacen más manipulables y flexibles las representaciones de los niños, cómo surge al acceso consciente al conocimiento".

Tanto Karmiloff-Smith (1994) como Gombert (1992) sugieren que el desarrollo metalingüístico se produce en tres fases: adquisición de habilidades iniciales, fase epilingüística (término referente a la recepción, Culioli, 1990) y fase metalingüística (relacionada con la producción lingüística). Los autores utilizan el concepto de fase porque éste, a diferencia del de etapa, propuesto por Piaget (1984), permite considerar cierta recursividad. En la teoría piagetiana, un niño sólo puede encontrarse en una etapa por vez. Luego de alcanzados los logros de una determinada etapa, ingresa a una nueva etapa en la que los contenidos de la realidad se representan de manera distinta. De este modo, el desarrollo es visto como una escala en la que el niño va cambiando cualitativamente sus operaciones cognitivas, volviéndose más eficiente para interactuar con la realidad. En cambio, en el modelo de fases, no es necesario dejar una fase para pasar a la otra, se puede operar en fases diferentes a la vez, en diversos microdominios y en situaciones distintas. En el caso concreto de la metalingüística, las dos primeras fases (la fase de las habilidades iniciales y la epilingüística) ocurren de manera más o menos sistemática, mientras que la tercera (la metalingüística) depende del contexto. 
En la adquisición de las habilidades lingüísticas iniciales, el niño aprende pares unifuncionales, en los que una forma lingüística corresponde a un determinado contexto. Halliday (1975) señala una etapa inicial muy similar en su enfoque funcionalista de la adquisición del lenguaje. Sin embargo, en la versión de Karmiloff-Smith (1994), dicho proceso no es guiado únicamente por el uso sino que posee pre-programaciones innatas de las habilidades lingüísticas de base y aprende los pares funcionales gracias a la mediación del modelo lingüístico presente en el entorno.

A medida que el niño va adquiriendo más su lengua materna, el pequeño se da cuenta de que hay más de una manera de referirse a un contexto, por esto, debe abandonar los pares funcionales. Esto obliga al niño a entrar en un proceso de reorganización que es característico de la segunda fase, en la que se produce la adquisición del dominio epilingüístico, razón por la cual los conocimientos implícitos acumulados dentro de la primera fase se reorganizan en la memoria a largo plazo. A partir de allí los pares forma-función son sustituidos por relaciones multifuncionales, pero el niño las usa mediante ajustes automáticos y no puede explicitarlas todavía en forma verbal.

Puyuelo et al. (2003) señalan dos diferencias fundamentales entre la visión de Gombert (1992) y la de Karmiloff-Smith (1994). Esta última sostiene que en la etapa epilingüística se reorganiza la información de la fase anterior (adquisición). En cambio, el autor francés plantea que esta reorganización surge de la necesidad de interrelacionar los conocimientos de la primera fase, con otros, recientemente descubiertos, acerca de las mismas formas lingüísticas o acerca de otras formas que están asociadas con ellas y están en curso de apropiación. Otra diferencia apunta a la naturaleza de la información que se está manejando en esta etapa. Mientras que para Karmiloff-Smith, en esta fase, la reorganización es impermeable a las influencias externas, Gombert afirma que, si bien la función principal de esta fase es una articulación interna de los conocimientos implícitos, lo que permitirá al sujeto el dominio funcional no conciente del sistema, la elaboración de las reglas que rigen el uso de las formas lingüísticas viene determinada por lo que el niño descubre acerca de dichas reglas en los funcionamientos lingüísticos en situación.

En último lugar, la fase metalingüística no sólo se relaciona con la capacidad de reflexionar y autorregular el propio lenguaje, sino también con la posibilidad de explicitar esta conciencia que se posee del propio código. Esta fase no ocurre de forma totalmente sistemática y homogénea. Los hablantes suelen ser más autorreflexivos en algunos aspectos del lenguaje que en otros, y esto parece depender de los niveles de la lengua y de las características de cada sujeto (Karmiloff-Smith 1994). En este sentido, Gombert (1992) analiza las habilidades metalingüísticas en los diversos niveles de la lengua, por lo que dichas habilidades se agrupan en la conciencia metafonética, metasemántica, metasintáctica, metatextual y metapragmática.

Ahora bien, de todos estos aspectos, en el presente trabajo se examina la conciencia metapragmática. Gombert (1992) y Verschueren (2000) la ubican en el uso expresivo y comprensivo del lenguaje (especialmente oral) y la definen no sólo como un saber acerca de las relaciones entre contexto y mensaje lingüístico, sino también como conciencia y autocontrol de la comprensión y producción lingüística.

Partiendo de lo definido a nivel metalingüístico, en general, Gombert (1992) considera que los niños usuarios de una lengua adquieren la conciencia metapragmática en fases distintas. La de adquisición inicial se caracteriza porque en ella el niño utiliza las primeras formas en ciertos contextos. En la epipragmática, el niño no discrimina explícitamente entre mensaje lingüístico y mensaje contextual, pero realiza ajustes automáticos entre ambos tipos de información, cometiendo algunos errores de interpretación cuando dicha relación no es literal. Por último, la fase metapragmática se inicia alrededor de los seis años, puesto que se adquiere conciencia de la diferencia entre lo lingüístico y lo contextual como también se adquiere control de esta relación en uso. En este caso, el sujeto es capaz de explicitar las relaciones entre la forma lingüística y el contexto, en la producción e interpretación de los mensajes lingüísticos. Para Gombert, el 
acceso a esta fase está muy relacionado con el aumento en la capacidad de la memoria operativa. Por este motivo, la habilidad metapragmática no se aplica en forma sistemática a todas las situaciones, sino que depende de la complejidad tanto del mensaje lingüístico como del medio contextual en el que éste tiene lugar y con el que se relaciona.

En adición, es necesario acudir a la visión de Verschueren (2000) respecto de cuáles serían las señales que le permitirían al hablante de una lengua ajustar lo lingüístico con lo no lingüístico, cuando se autorregula. Además, para este autor los indicadores de conciencia metapragmática funcionan de dos modos en el lenguaje en uso: como mecanismos de anclaje que localizan formas lingüísticas en relación con el contexto, y como señales o marcadores de interpretaciones reflexivas de los usuarios del lenguaje de las actividades que realizan.

\section{Método}

\section{Sujetos}

Para observar el desarrollo ontogenético de la conciencia metapragmática se aplicó la prueba de conciencia metapragmática [CMP] a una muestra de 160 alumnos de distintos cursos de enseñanza básica, pertenecientes a dos escuelas particularessubvencionadas de las ciudades de Valparaíso y Quilpué. Los sujetos de la muestra se distribuyen como se muestra en el Tabla 1.

TABLA 1

Número de alumnos en la muestra, según curso y género

\begin{tabular}{|c|c|c|c|c|}
\hline \multirow{2}{*}{ Curso } & \multirow{2}{*}{$\begin{array}{c}\text { Años } \\
\text { de edad } \\
\text { promedio }\end{array}$} & \multicolumn{2}{|c|}{ Género } & \multirow{2}{*}{ Total } \\
\hline & & Femenino & Masculino & \\
\hline 1o Básico & 6 & 17 & 23 & 40 \\
\hline 3o Básico & 8 & 9 & 31 & 40 \\
\hline 5o Básico & 10 & 9 & 31 & 40 \\
\hline 7º Básico & 12 & 7 & 33 & 40 \\
\hline
\end{tabular}

Fuente: elaboración propia.
Como puede verse la muestra incluye niños y niñas de entre 6 y 12 años, pero no se incluyen sujetos de todas de las edades intermedias, sino que se consideran menores cuyas edades se escalan año de por medio $(6,8,10,12)$. ¿Por qué se toma esta decisión metodológica? En otras mediciones de desarrollo del lenguaje en la edad escolar (Crespo, 2008; Crespo, Benítez \& Cáceres 2007; Crespo \& Cáceres, 2006) se encontró un solapamiento entre edades próximas ( 6 y 7,8 y 9,10 y 11$)$. Por ese motivo se creyó oportuno considerar los grupos distanciados por dos años en la edad, para observar las diferencias con mayor claridad. Esto no obsta para que más adelante se puedan hacer observaciones con todas las edades, con el fin de precisar posibles momentos críticos de este desarrollo. Por otra parte, cada grupo de edad estuvo compuesto por 40 niños y niñas, número mínimo de participantes que se necesita para validar los análisis estadísticos.

\section{Instrumento}

El instrumento CMP es un test multimedia compuesto por 29 ítems mediante los cuales se evalúa la conciencia metapragmática. Los sujetos deben dar cuenta de los evidentes desajustes entre el contexto y el mensaje lingüístico. Los 29 ítems están organizados en tres tipos de ejercicio: Referente Ambiguo, Instrucciones Ambiguas y Diálogo Observado, todas situaciones estereotípicas en las que se manifiesta la conciencia metapragmática (Gombert, 1992; Flavell, 1993). El instrumento multimedia CMP se aplica en forma individual a cada niño con el asesoramiento de un evaluador que consigna cada una de sus respuestas.

Los ítems de Referente Ambiguo corresponden a un enunciado referencial en el que se presenta un deíctico que no especifica de manera clara su referente. Por ejemplo, se proyecta la imagen de cuatro gatos, dos de los cuales usan un collar. Luego se solicita al niño que identifique el gato con collar. El uso del artículo definido singular llevaría al sujeto a pensar que existe un solo individuo con ese rasgo, cuando el contexto presenta dos. Ante esta situación de ambigüedad, el niño en evaluación 
tendrá tres respuestas posibles: elegir cualquiera de los dos gatos con collar, lo cual implica que no es capaz de darse cuenta de la ambigüedad; manifestar su duda, pero no saber cómo explicarla, lo cual implica que hay un desarrollo incipiente de la conciencia metapragmática o manifestar su duda y explicar cuál es la inadecuación entre contexto y expresión lingüística en ese caso. Las dos primeras respuestas se consideran epipragmáticas, con 0 y 1 punto respectivamente y a la tercera se la rotula como metapragmática, 2 (dos) puntos.

Los ítems de Instrucciones Ambiguas consisten en la entrega de instrucciones erróneas o incompletas para participar en algún juego o realizar una acción. Este tipo de ítems ha sido utilizado por Flavell (1993) con anterioridad. En esta clase de ejercicios, el niño en evaluación también tiene tres opciones: empieza a jugar hasta que se da cuenta que algo anda mal o manifiesta su duda, pero no sabe explicarla, permaneciendo dentro de una esfera epipragmática; o explica su duda, dando cuenta de la ambigüedad y demostrando una capacidad más metapragmática. Nuevamente las respuestas se valoran 0 (cero), 1 (uno) ó 2 (dos) puntos.

Por último, los ítems de Diálogo Observado ilustran la observación de una situación de un malentendido entre dos personas debido a la falta de precisión del emisor del enunciado. En este caso, el niño en evaluación debe explicar de quién ha sido la culpa del malentendido. Por ejemplo, un hijo deja una flor para su madre sobre la mesa y luego se lo comunica, pero, cuando la madre va en busca de dicha flor, se encuentra con dos flores sobre la mesa y escoge la flor equivocada. En este caso el niño tiene tres posibles respuestas de las cuales dependerá el puntaje asignado. Por un lado, puede señalar que la madre ha elegido la flor correcta, o indicar que la madre no ha elegido la flor correcta, pero que el error ha sido de ella, ambas respuestas se consideran epipragmáticas y reciben 0 y 1 punto respectivamente; por otro, puede puntualizar que la madre se ha equivocado debido al enunciado ambiguo de su hijo, lo cual implica una respuesta metapragmática valorada en 2 (dos) puntos.

En el Tabla 2 se presenta la distribución de los ítems por tipo de ejercicio y los puntajes máximos.
TABLA 2

Número de ítems de respuesta, por tipo de ejercicio y puntaje máximo

\begin{tabular}{lcc}
\hline \multicolumn{1}{c}{ Pregunta } & № Preguntas & $\begin{array}{c}\text { Puntaje } \\
\text { Máximo }\end{array}$ \\
\hline Referente Ambiguo & 10 & 20 \\
Instrucciones Ambiguas & 9 & 18 \\
Diálogo Observado & 10 & 20 \\
TOTAL & 29 & 58 \\
\hline
\end{tabular}

Fuente: elaboración propia.

Cabe señalar que la selección de un diseño multimedia de dibujos animados obedece a que este tipo de instrumento permitiría simular de manera más adecuada una situación de oralidad real, dando simultáneamente estímulos visuales y lingüísticos. Su ventaja, frente a instrumentos que utilizan láminas e información lingüística oral o lápiz y papel, es evidente, ya que estos últimos exigen mayor atención y un esfuerzo adicional en la representación. Por tanto, consideramos que el diseño multimedia permite reflejar de manera adecuada el desarrollo de la conciencia metapragmática.

Otra ventaja del formato empleado para la elaboración de este instrumento, consiste en que el creador del software es quien decide qué opciones de participación le dará al usuario, lo cual permite unificar de manera más sistemática la información recopilada (Bedoya, 1997). Finalmente, cabe señalar que el test CMP es un instrumento altamente confiable para medir conciencia metapragmática en niños de ocho años, de acuerdo a los resultados de las pruebas estadísticas presentadas por Crespo (2009), ya que dio un Coeficiente del Alpha de Cronbach (1951) de 0,89. El hecho de que la CMP sea altamente confiable implica que tiene un error estándar de medición reducida (García-Garro, Ramos-Ortega, León-Ponce \& Olivera-Chávez, 2007). Por lo anterior, se puede considerar que se cuenta con un instrumento de medición óptimo, para llevar a cabo otras investigaciones del tema. 


\section{Análisis de los resultados}

En esta sección se presentarán, primero, los porcentajes generales de los tipos de respuesta que los niños dieron en la prueba; seguidamente, se examinarán las respuestas a los distintos ítems; y, por último, se discutirá la fundamentación de las diferencias significativas ${ }^{1}$.

\section{Porcentajes en general por tipo de respuesta}

Ya se señaló en la descripción del instrumento que las respuestas posibles podían ser consideradas como epipragmáticas ( 0 y 1 punto) o como metapragmáticas ( 2 puntos). En un primer análisis descriptivo de las respuestas de los niños, se observaron los porcentajes de respuestas que dieron todos los individuos, es decir, 40 sujetos por grupo de edad respondieron 29 ítems dando un total de 1160 respuestas posibles; sobre ese total se consideró la proporción de respuestas epipragmáticas y metapragmáticas. La Tabla 3 ilustra este hecho.

TABLA 3

Porcentaje de respuestas obtenidas, según curso

\begin{tabular}{|c|c|c|c|c|}
\hline \multirow[b]{2}{*}{ Curso } & \multicolumn{4}{|c|}{ Resultados } \\
\hline & $\begin{array}{l}\text { Respues- } \\
\text { tas } \\
\text { Epiprag- } \\
\text { máticas }\end{array}$ & $\begin{array}{l}\text { Porcen- } \\
\text { taje } \\
\text { Epiprag- } \\
\text { mático }\end{array}$ & $\begin{array}{l}\text { Respues- } \\
\text { tas } \\
\text { Metaprag- } \\
\text { máticas }\end{array}$ & $\begin{array}{l}\text { Porcen- } \\
\text { taje } \\
\text { Metaprag- } \\
\text { mático }\end{array}$ \\
\hline $\begin{array}{c}1^{\mathrm{o}} \\
\text { Básico }\end{array}$ & 951 & $82 \%$ & 209 & $18 \%$ \\
\hline $\begin{array}{c}\text { 3o } \\
\text { Básico }\end{array}$ & 788 & $68 \%$ & 372 & $33 \%$ \\
\hline $\begin{array}{c}5^{\mathrm{o}} \\
\text { Básico }\end{array}$ & 545 & $47 \%$ & 615 & $53 \%$ \\
\hline $\begin{array}{c}7^{\circ} \stackrel{ }{ } \\
\text { Básico }\end{array}$ & 336 & $29 \%$ & 824 & $71 \%$ \\
\hline
\end{tabular}

Fuente: elaboración propia.

1 Se agradece a Carlos Montenegro y a Lorena Pérez por su tratamiento estadístico de la información que permitió arribar a estos resultados.
Como puede verse, todos los participantes dan los dos tipos de respuestas. Sin embargo, el porcentaje de las epipragmáticas disminuye y el de las metapragmáticas aumenta a medida que se incrementa la edad de los sujetos. En otras palabras, a medida que crecen los niños parecen utilizar más las respuestas metapragmáticas que las epipragmáticas.

Como ya se ha señalado al describir el instrumento, la respuesta epipragmática es aquella en la cual el sujeto no manifiesta de manera evidente que se ha dado cuenta del un cierto desajuste entre el mensaje lingüístico y el contexto, mientras que en la metapragmática, sí es capaz de verbalizar esa incongruencia. Como puede verse el paso a esta capacidad de dar cuenta del desajuste entre mensaje lingüístico y contexto extralingüístico, es gradual y se va dando de manera más evidente con la edad.

\section{Resultados por ítem y tipo de respuesta}

Ya se señaló que el instrumento CMP consta de 29 ítems clasificados en los siguientes 3 tipos de preguntas: Diálogo Observado, Instrucciones Ambiguas y Referente Ambiguo. Los resultados señalaron que no en todas ellas se produjo del mismo modo el incremento de conciencia metapragmática a medida que aumentaba la edad.

Como se observa en el Figura 1, el decrecimiento de respuestas epipragmáticas y el incremento de las metapragmáticas se daban con mucha claridad a medida que aumentaba la edad de los sujetos en los ítems de Instrucciones Ambiguas, preguntas en las cuales se le instruía al niño para realizar una tarea pero de manera ambigua (saltando algún paso, refiriendo objetos ausentes o señalando dos posibles ganadores si era un juego). En las respuestas epipragmáticas el niño trataba de seguir las instrucciones o era incapaz de verbalizar que había algo poco claro, mientras que en la respuesta metapragmática indicaba verbalmente la causa de su confusión. Las respuestas epipragmáticas que dan los sujetos de 12 años en este ítem, llegan a la misma proporción que las metapragmáticas que dieron los de seis años. Y, análogamente, la proporción de respuestas metapragmáticas de los 

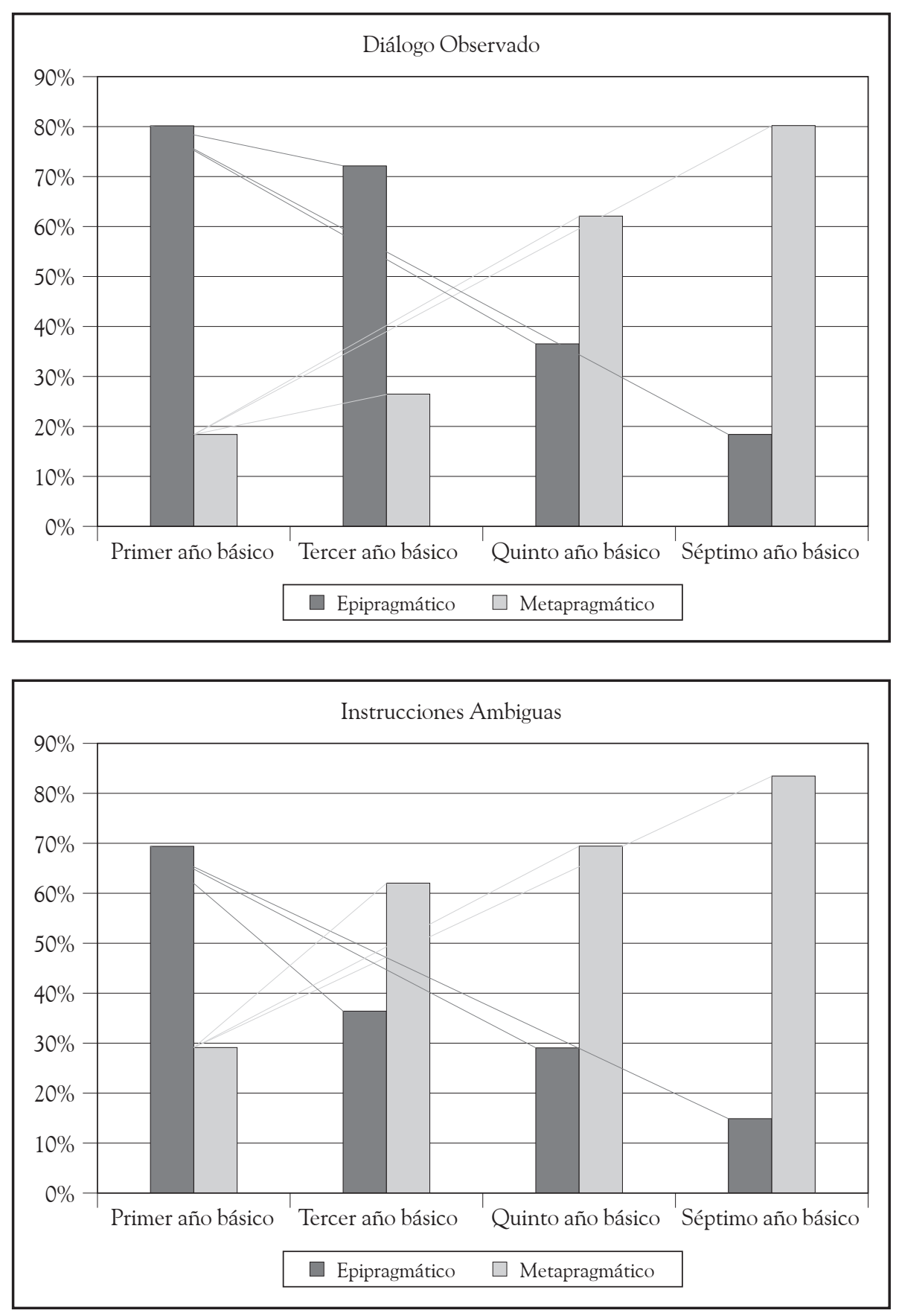

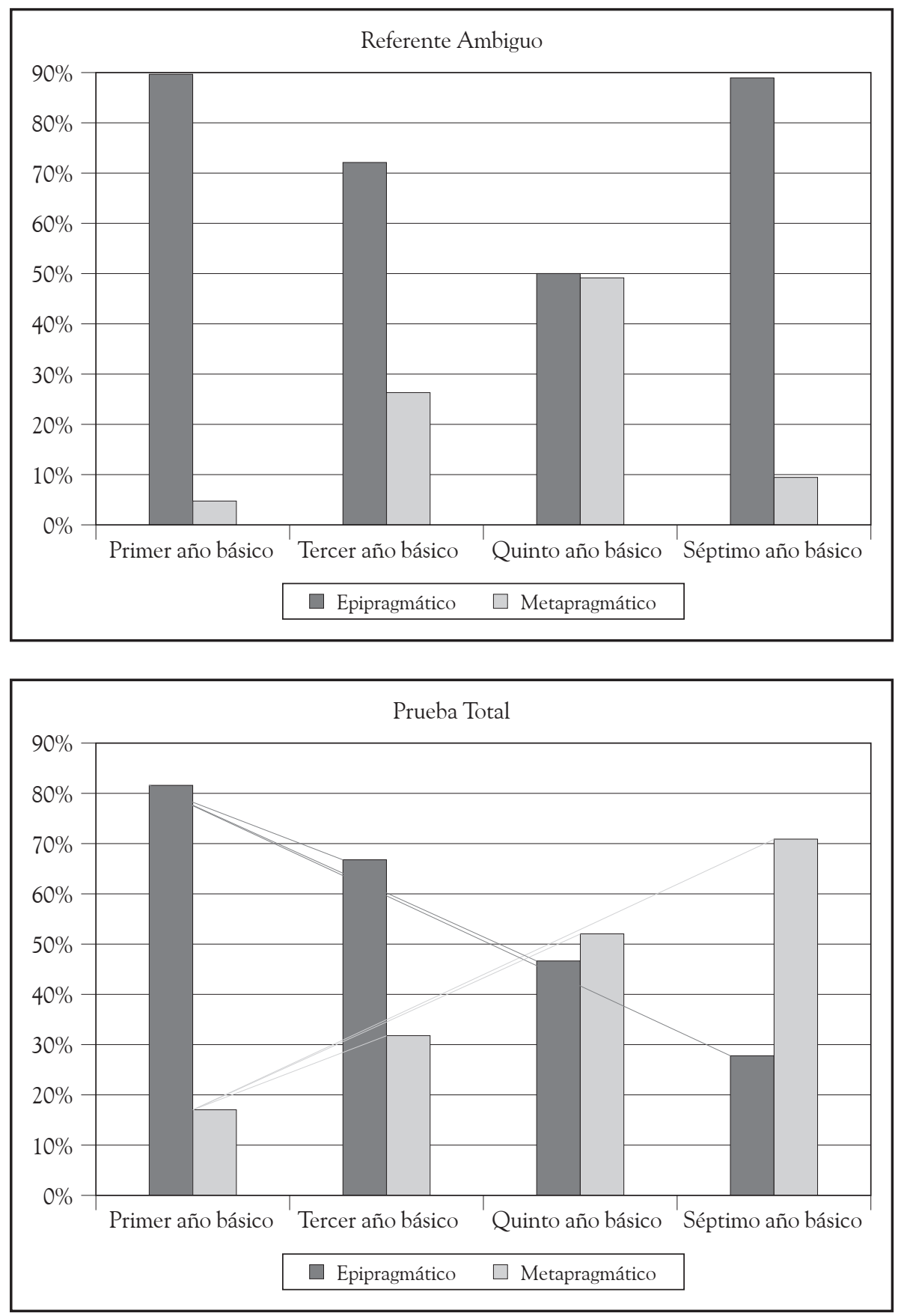

Figura 1

Porcentaje de respuestas obtenidas, según curso y tipo de pregunta.

Fuente: elaboración propia. 
sujetos evaluados igualó a la proporción de las respuestas epipragmáticas de los niños de seis. De este modo, se observa que mientras los sujetos van respondiendo de un modo más reflexivo, van abandonando sus respuestas más automáticas.

En el ejercicio de Diálogo Observado se da el mismo comportamiento en los porcentajes, solo que un poco menos armónico ya que los sujetos de 12 años producen más respuestas metapragmáticas (85\%) que las respuestas epipragmáticas de los niños de menor edad (70\%). Como se señaló al presentar el instrumento, este ejercicio implicaba la observación de una situación de un malentendido entre dos personas debido a la falta de precisión del emisor del enunciado. En este caso, el niño en evaluación debe identificar el malentendido y explicar de quién ha sido la culpa del malentendido.

Esta tendencia se rompe en el ítem de Referente Ambiguo. Recuérdese, como se explicó al presentar el instrumento, que ítems de este tipo corresponden a un enunciado referencial en el que se presenta un deíctico que no especifica de manera clara su referente. Por ejemplo, se proyecta la imagen de cuatro gatos, dos de los cuales usan un collar. Luego se solicita al niño que identifique el gato con collar. El uso del artículo definido singular llevaría al sujeto a pensar que existe un solo individuo con ese rasgo, cuando el contexto presenta dos. Ante esta situación de ambigüedad, el niño en evaluación tendrá dos formas de respuestas: epipragmáticas (el niño no verbaliza la incoherencia) y metapragmáticas (el niño verbaliza la incoherencia). Como puede verse en el gráfico, la curva decrecimiento epipragmático-incremento metapragmático sufre un cambio abrupto con los sujetos de 12 años, que no llegan a un 10\% de respuestas metapragmáticas, mientras que exhiben casi un 90\% de respuestas epipragmáticas, siendo sus logros iguales en proporción a los niños de menor edad. El fenómeno de los resultados observados en Referente Ambiguo parece tener su explicación en una sobreinterpretación por parte de los sujetos mayores. Estos consideraron que la respuesta del ejercicio era "demasiado evidente” y que debía haber algún truco o engaño, es el motivo por el cual prefirieron dar respuestas más epipragmáticas.
Más adelante se argumentará que el hecho de que la relación epipragmática /metapragmática se dé de manera distinta en los diferentes ítems, explica mejor la naturaleza de la variable en estudio.

\section{Fundamentación de las diferencias significativas}

En el apartado anterior, se observó que las respuestas metapragmáticas aumentan mientras disminuyen las epipragmáticas a medida que los sujetos son mayores. Sin embargo, este hallazgo es puramente descriptivo y no fundamenta el desarrollo ontogenético de la conciencia metapragmática. Es necesario establecer estadísticamente si estas diferencias de puntaje entre los grupos son significativas. Con el objetivo de demostrar esto, se utilizó un modelo lineal generalizado de efectos mixtos [GLMM] (McCulloch \& Searle, 2001). Este análisis ha permitido considerar a todos los niños como una muestra aleatoria y al curso (indicador de la edad) como un factor fijo.

Posteriormente, se procedió a comparar los cursos adyacentes ( $1^{\circ}-3^{\circ}-3^{\circ}-5^{\circ}$ y $\left.5^{\circ}-7^{\circ}\right)$, comprobando que efectivamente existe una diferencia significativa ascendente en el nivel metapragmático conforme aumenta el curso. De esta forma, el ajuste del modelo GLMM verificó que existen diferencias significativas en los niveles de conciencia metapragmática entre los niños de primero, tercero, quinto y séptimo básico.

También se realizaron 6 pruebas de hipótesis para comprobar si existen diferencias significativas en los niveles de conciencia metapragmática de los niños de primero, tercero, quinto y séptimo básico. Las hipótesis comprobadas fueron:

HO: La proporción de respuestas metapragmáticas en alumnos es igual entre cursos de enseñanza básica.

H1: La proporción de respuestas metapragmáticas en alumnos es distinta entre cursos de enseñanza básica.

Para aceptar la hipótesis $\mathrm{H} 1$ y rechazar la HO, la estadística de prueba debe superar el valor crítico 
1.96, correspondiente al valor tabulado de una distribución normal estandarizada que utiliza un nivel de significancia o probabilidad de cometer error de tipo I de 5\%. Los resultados se presentan en el Tabla 4.

TABLA 4

Estadísticas de pruebas de curso

\begin{tabular}{cccc}
\hline $\begin{array}{c}\text { Estadística de } \\
\text { Prueba }\end{array}$ & $\begin{array}{c}\mathbf{1}^{\mathbf{o}} \text { año } \\
\text { básico }\end{array}$ & $\begin{array}{c}\text { 3o año } \\
\text { básico }\end{array}$ & $\begin{array}{c}\mathbf{5}^{\mathbf{o}} \text { año } \\
\text { básico }\end{array}$ \\
\hline $1^{\text {o }}$ año básico & $8,13^{* *}$ & $17,56 * *$ & $25,78^{* *}$ \\
3o año básico & & $9,82^{* *}$ & $18,57^{* *}$ \\
5o año básico & & & $9,1^{* *}$ \\
\hline
\end{tabular}

Fuente: elaboración propia.

Como se observa en el Tabla 4, todos los valores de las estadísticas de prueba son mayores que el valor crítico $(1,96)$. Con esto se rechazaron las hipótesis nulas de igualdad de proporciones entre los distintos cursos. Una vez más quedó comprobada la significatividad en la diferencias de puntaje que muestran los cursos.

\section{Conclusión}

Uno de los hechos que queda evidenciado al comparar los resultados de las respuestas epigramáticas y las metapragmáticas, consiste en que todos los niños evaluados manifiestan respuestas epipragmáticas y metapragmáticas. Las diferencias entre los grupos no consisten en que los más pequeños elijan las respuestas más automáticas y, a partir de cierta edad, prefieran otras más reflexivas, sino en que el porcentaje de las epipragmáticas disminuye y el de las metapragmáticas aumenta gradualmente a medida que se incrementa la edad de los niños. Este hecho parece confirmar la visión de KarmiloffSmith (1994) y Gombert (1992) quienes plantean que el desarrollo metapragmático tiene lugar en fases recursivas que el sujeto recorre simultáneamente. Además, se cuestiona en parte la visión de autores como van Kleeck (1994), que consideran el desarrollo de estos procesos en etapas sucesivas, utilizando una interpretación más piagetiana.

Por otro lado, los sujetos revelaron no tener la misma dificultad para todos los ítems. Este hecho se explica porque las relaciones entre contexto y mensaje lingüístico no siempre se dan de la misma manera, siendo entonces posible para el niño dar cuenta de unas relaciones más fácilmente que de otras. En este punto, parecen corroborarse las impresiones de Gombert (1992), en el sentido que la habilidad metapragmática no se aplica en forma sistemática a todas las situaciones, sino que depende de la forma en que se relacionen las informaciones de distintas fuentes y la posición que el interlocutor ocupa respecto a ellas, o - como lo señala Verschueren (2002) — las diferencias en su grado de saliencia.

En último lugar, queda considerar las diferencias significativas observadas en los puntajes en conciencia metapragmática que demostraron los sujetos a medida que aumentaba la edad. Este hecho permite, por una parte, apreciar un desarrollo de la capacidad de autorregular el uso del lenguaje oral durante la edad escolar y, por otra, concluir que este fenómeno ocurre con cierta regularidad, sin grandes saltos ni edades críticas. Este estudio inicial permite, en parte, describir el desarrollo de una habilidad que ocurre en forma concomitante con el desarrollo tardío del lenguaje oral. Sólo resta señalar cómo se relaciona con él y en qué medida sustenta o se relaciona el desarrollo de la lectoescritura de los niños en los primeros años escolares. Considerar esta variable permitirá en el futuro visualizar nuevas posibilidades para estimular el desarrollo del lenguaje oral infantil.

\section{Referencias}

Bedoya, A. (1997, septiembre). ¿Qué es la interactividad? Revista Electrónica sinpapel.com. Consultado el 25 febrero, 2003, en http://www.sinpapel.com/ art001.shtml

Bialystok , E. (1992). Attentional control in children's metalinguistic performance and measures of field 
independence. Developmental Psychology, 28(4), 654-664.

Crespo, N. (2008). Los actos de habla indirectos en escolares: aprendiendo a comprender lo que se quiere decir. Revista de Fonoaudiología, 2, 23-35.

Crespo, N. (en prensa). La medición de la conciencia metapragmática de los niños: Resolviendo la ambigüedad en la comprensión oral. Revista de Lingüística Teórica y Aplicada.

Crespo, N., Benítez, R. \& Cáceres, P. (2007). La comprensión de las ironías orales: el desarrollo de una habilidad. Estudios Filológicos, 42, 79-94.

Crespo, N. \& Cáceres, P. (2006). La comprensión oral de las frases hechas: un fenómeno de desarrollo tardío del lenguaje. Revista de Lingüística Teórica y Aplicada, 44(2), 77-90.

Cronbach, L. J. (1951). Coefficient alpha and the internal structure of tests. Psychometrika, 16(3), 297-334

Culioli, A. (1990). Pour une linguistique de l'énonciation. Opérations et représentations. Paris: Ophrys.

Flavell, J. (1993). El desarrollo cognitivo. Madrid: Aprendizaje Visor.

Flórez-Romero, R., Torrado-Pacheco, M. C. E. \& Magnolia Mesa, C. (2006). Emergencia de las capacidades metalingüísticas. Revista Latinoamericana de Psicología, 38(3), 457-475.

García-Garro, A. J., Ramos-Ortega, G., León-Ponce, M. A. \& Olivera-Chávez, A. (2007). Instrumentos de evaluación. Revista Mexicana de Anestesia, 30(3), 158-164.

Gombert, J. (1992). Metalinguistic Development. Chicago: University of Chicago Press.

Halliday, M. A. K. (1975). Learning how to mean: Explorations in the development of language. London: Edward Arnold.
Jakobson, R. (1963). Essais de linguistique générale. Paris: Minuit.

Karmiloff-Smith, A. (1994). Más allá de la modularidad. Madrid: Alianza.

Levy, Y. (1999). Early metalinguistic competence: Speech monitoring and repair behavior. Developmental Psychology, 35 (3), 822-834.

McCulloch, C. E. \& Searle, S. R. (2001). Generalized, Linear, and Mixed Models. New York: Wiley.

Nippold, M. (1998). Later language development. Austin, TX: Pro. Ed.

Piaget, J. (1984). Psicología del niño. Madrid: Morata.

Piaget, J. (1987). La formación del símbolo en el niño: imitación, juego, imagen y representación. México: Fondo de Cultura Económica.

Puyuelo, M. \& Rondal, J. A. (2003). Manual de desarrollo y alteraciones del lenguaje: aspectos evolutivos y patologías en el niño y en el adulto. Madrid: Masson.

Ravid, D. \& Tolchinsky, L. (2002) Developing linguistic literacy: A comprehensive model. Child Language, 29, 417-447.

van Kleeck, A. (1994). Metalinguistic development. In G. Wallach \& K. Butler (Eds.), Language learning disabilities in school-age children and adolescents: Some principles and applications (pp. 53-98). New York: Macmillan.

van Kleeck, A. (1995). Emphasizing form and meaning separately in rereading and early reading instruction. Topics in Language Disorders, 16, 27-49.

Verschueren, J. (2000). Notes on the role of metapragmatic awareness. Language use, Pragamtics, 10(4), 439-456.

Verschueren, J. (2002). Para entender la Pragmática. Madrid: Gredos. 